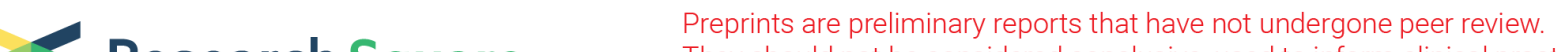 $\begin{array}{ll}\text { Research Square } & \text { They should not be considered conclusive, used to inform clinical practice, } \\ \text { or referenced by the media as validated information. }\end{array}$
}

\section{Acute SARS-CoV-2 Infection is Highly Cytopathic, Elicits a Robust Innate Immune Response and is Efficiently Prevented by EIDD-2801}

\section{Angela Wahl}

International Center for the Advancement of Translational Science, Division of Infectious Diseases, Department of Medicine, and Center for AIDS Research, University of North Carolina at Chapel Hill

Lisa Gralinski

UNC

\section{Claire Johnson}

University of North Carolina-Chapel Hill

\section{Wenbo Yao}

University of North Carolina-Chapel Hill

\section{Martina Kovarova}

University of North Carolina-Chapel Hill

\section{Kenneth Dinnon}

University of North Carolina at Chapel Hill https://orcid.org/0000-0002-8942-1551

\section{Hongwei Liu}

University of North Carolina-Chapel Hill

\section{Victoria Madden}

University of North Carolina https://orcid.org/0000-0002-7909-7743

\section{Halina Krzystek}

University of North Carolina-Chapel Hill

\section{Chandrav De}

International Center for the Advancement of Translational Science, Division of Infectious Diseases, Department of Medicine, and Center for AIDS Research, University of North Carolina at Chapel Hill

\section{Kristen White}

University of North Carolina-Chapel Hill

\section{Alexandra Schäfer}

University of North Carolina at Chapel Hill

\section{Tanzila Zaman}

University of North Carolina-Chapel Hill

\section{Sarah Leist}

University of North Carolina at Chapel Hill

\section{Paul Grant}


University of North Carolina-Chapel Hill

\section{Kendra Gully}

University of North Carolina at Chapel Hill

\section{Frederic Askin}

University of North Carolina-Chapel Hill

\section{Edward Browne}

UNC HIV Cure Center, Division of Infectious Diseases, Department of Medicine, and Center for AIDS Research, University of North Carolina

\section{Corbin Jones}

University of North Carolina at Chapel Hill

\section{Raymond Pickles}

University of North Carolina-Chapel Hill

\section{Ralph Baric}

University of North Carolina https://orcid.org/0000-0001-6827-8701

\section{J Victor Garcia ( $\square$ victor_garcia@med.unc.edu )}

University of North Carolina at Chapel Hill https://orcid.org/0000-0003-3726-0482

\section{Biological Sciences - Article}

Keywords: COVID-19, SARS-CoV-2, virus replication, endogenous bat coronaviruses,

Posted Date: September 24th, 2020

DOI: https://doi.org/10.21203/rs.3.rs-80404/v1

License: (c) (1) This work is licensed under a Creative Commons Attribution 4.0 International License. Read Full License

Version of Record: A version of this preprint was published at Nature on February 9th, 2021. See the published version at https://doi.org/10.1038/s41586-021-03312-w. 


\section{Abstract}

All known recently emerged human coronaviruses likely originated in bats. Here, we used a single experimental platform based on human lung-only mice (LoM) to demonstrate efficient in vivo replication of all recently emerged human coronaviruses (SARS-CoV, MERS-CoV, SARS-CoV-2) and two highly relevant endogenous pre-pandemic SARS-like bat coronaviruses. Virus replication in this model occurs in bona fide human lung tissue and does not require any type of adaptation of the virus or the host. Our results indicate that bats harbor endogenous coronaviruses capable of direct transmission into humans. Further detailed analysis of pandemic SARS-CoV-2 in vivo infection of LoM human lung tissue showed predominant infection of human lung epithelial cells, including type II pneumocytes present in alveoli and ciliated airway cells. Acute SARS-CoV-2 infection was highly cytopathic and induced a robust and sustained Type I interferon and inflammatory cytokine/chemokine response. Finally, we evaluated a preexposure prophylaxis strategy for coronavirus infection. Our results show that prophylactic administration of EIDD-2801, an oral broad spectrum antiviral currently in phase II clinical trials for the treatment of COVID-19, dramatically prevented SARS-CoV-2 infection in vivo and thus has significant potential for the prevention and treatment of COVID-19.

\section{Main Text}

The recently emerged human pandemic coronavirus Severe Acute Respiratory Syndrome coronavirus-2 (SARS-CoV-2), the causative agent of coronavirus disease (COVID)-19, has spread to six continents resulting in substantial morbidity and mortality worldwide ${ }^{1}$. Bats serve as a natural reservoir for coronaviruses and are the presumed source of SARS-CoV-2 and the highly pathogenic human coronaviruses SARS-CoV and Middle East Respiratory Syndrome (MERS)-CoV ${ }^{2}$. Transmission of coronaviruses from bats to other species is well-documented and adaptation in an intermediary host can facilitate their transmission to humans ${ }^{2}$. While it is possible that SARS-CoV-2 was transmitted to humans via an intermediate host such as pangolins, phylogenic analysis indicates that the SARS-CoV-2 lineage has circulated in bats for decades and evolved in bats into a virus capable of replicating in human cells ${ }^{3}$. Thus, bats are a potential reservoir for coronaviruses with human pandemic potential that can be directly transmitted to humans. Given the repeated and accelerating emergence of highly pathogenic coronaviruses, it has become increasingly important to monitor and characterize coronaviruses circulating in bats and to identify the viral determinants of human infection, disease, and global spread as well as to develop effective therapeutic interventions. Animal models are useful in studying highly pathogenic human coronaviruses, the emergence potential of zoonotic coronaviruses, and to evaluate novel inhibitors for their ability to control coronavirus infection ${ }^{4-15}$. However, human coronaviruses do not replicate in mice without either extensive adaptation of the virus or genetic modification of the host by genetic editing of the receptor or by introducing the individual human receptor genes for each virus ${ }^{4-}$ $11,14,15$. Although existing rodent models have made several important contributions to our understanding of coronavirus infection and pathogenesis, none of these models possess the diverse set of primary human cells present in the human lung that can serve as targets for viral infection ${ }^{16}$. Here, we show that 
human lung-only mice (LoM), immune deficient mice implanted with authentic human lung tissue ${ }^{17}$, allow for the in vivo study of all recently emerged human coronaviruses (SARS-CoV, MERS-CoV, SARSCoV-2) in a single platform that permits direct comparison of experimental outcomes. Using LoM, we also established that bats harbor novel coronaviruses capable of efficient replication in human lungs without prior adaptation. In addition, we performed an in-depth in vivo analysis of acute SARS-CoV-2 infection in the human lung. Our results revealed robust SARS-CoV-2 replication, pathogenesis and sustained activation of the innate host immune response. Finally, we used this platform for the in vivo evaluation of EIDD-2801, an orally administered broad spectrum antiviral currently in phase II clinical trials for COVID19 treatment, to prevent SARS- CoV-2 infection. Our results show that EIDD-2801 efficiently prevented SARS-CoV-2 infection in vivo strongly supporting its progression in clinical development for COVID-19.

\section{Human and bat coronavirus replication in LoM}

LoM are constructed by subcutaneous implantation of a small piece of human lung tissue into the back of immune deficient mice (Fig. 1a). Previously, we demonstrated that the human lung tissue expands to form a highly vascularized palpable implant ${ }^{17}$ (Fig. 1a). These lung implants contain human fibroblast, epithelial, endothelial, and mesenchymal cells that form highly relevant lung structures including cartilaginous and non-cartilaginous bronchial airways lined with ciliated and non-ciliated epithelium, alveolar sac structures, and extensive vasculature ${ }^{17}$ (Extended Data Fig. 1a,b). We also showed that the human lung tissue of LoMs supports replication of a diverse set of emerging and clinically relevant human pathogens including Zika virus, human cytomegalovirus, respiratory syncytial virus and MERS$\mathrm{CoV}^{17}$. Recently emerged human coronaviruses have used at least two different receptors to gain entry into host cells, human angiotensin converting enzyme-2 (ACE2) and dipeptidyl peptidase 4 (DPP4). SARSCoV and SARS-CoV-2 use human ACE2 as a receptor while MERS-CoV uses DPP4 ${ }^{18-22}$. These differences in receptor usage influence viral tropism, pathogenesis and disease progression ${ }^{23}$. Infection of LoM with MERS-CoV resulted in robust virus replication and infection of human epithelial, endothelial and mesenchymal cells in vivo ${ }^{17}$. These findings were consistent with the broad cellular distribution of its receptor, DPP4 ${ }^{24}$.

Here, we first evaluated the potential of LoM to serve as a single platform to study all known recently emerged human coronaviruses and the potential of endogenous bat coronaviruses for human emergence. We first confirmed that ACE2, the receptor for SARS-CoV and SARS-CoV- 2 in human cells, was present on the surface of human epithelial cells (cytokeratin 19+) in the human lung tissues of LoM (Extended Data Fig. 1c,d). Next, LoM were inoculated with recently emerged coronaviruses SARS-CoV, MERS-CoV, or SARS-CoV-2 (Extended Data Table 1). LoM supported replication of all these viruses in vivo. Specifically, SARS-CoV and SARS-CoV- 2 infection resulted in mean virus titers of $1.76 \times 10^{8}$ and $2.42 \times 10^{7}$ $\mathrm{PFU} / \mathrm{g}$ respectively at 2 days post-infection (Fig. 1b). Viral nucleoprotein antigen was abundantly observed in the human lung tissues of SARS-CoV and SARS-CoV-2 infected LoM (Extended Data Fig. 2a). Consistent with our previous results ${ }^{17}$, we also observed robust replication of MERS-CoV in the human 
lung tissues of LoM with mean titers of $4.79 \times 10^{8} \mathrm{PFU} / \mathrm{g}$ in LoM human lung tissues at 2 days postinfection (Fig. 1b) and abundant viral antigen (Extended Data Fig. 2a).

Pre-pandemic bat coronaviruses WIV1-CoV and SHC014-CoV have high sequence homology to SARS-CoV, use ACE2 to infect human cells, and grow modestly in primary human airway cultures on liquid interface ${ }^{4,5}$. LoM were inoculated with WIV1-CoV or SHC014-CoV and virus titers in human lung tissues measured 2 days post-infection (Extended Data Table 1). WIV1-CoV and SHC014-CoV efficiently replicated in the human lung tissue of LoM with mean titers of $1.58 \times 10^{7}$ and $1.48 \times 10^{7} \mathrm{PFU} / \mathrm{g}$, respectively (Fig. 1b) and viral antigen was readily detected in human lung tissues (Extended Data Fig. 2b). Collectively, these results demonstrate that LoM serve as single platform where all newly emerged coronaviruses SARS-CoV, MERS-CoV, and SARS-CoV-2 replicated efficiently in human lung tissue. Importantly, the fact that SARS-like bat coronaviruses WIV1-CoV and SHC014-CoV also replicated efficiently in the LoM platform indicates that bats harbor coronaviruses that are potentially capable of direct transmission to humans, thus bypassing the need for further adaptation in an intermediary host.

\section{SARS-CoV-2 replication in LoM}

Given the state of the current COVID-19 pandemic and the urgent need to develop therapeutic and preventative approaches to control and prevent infection, we evaluated replication of SARS-CoV-2 in LoM in detail. Human lung tissues of LoM were inoculated with SARS-CoV-2 and titers of replication competent virus determined 2, 6, and 14 days post-exposure (Fig. 1c, Extended Data Table 2). High titers of replication competent virus were noted at all time points although they were highest 2 days postinfection (Fig. 1d). The distribution of virus-infected cells was determined with RNAscope (viral RNA) and immunofluorescence microscopy (viral nucleoprotein). Virus infection was widely distributed throughout the tissue with large numbers of cells positive for viral RNA (Fig. 1e) and nucleoprotein (Fig. 1f). Costaining with a human cytokeratin 19 antibody demonstrated that SARS-CoV-2 predominantly infects human epithelial cells in the lung (Fig. 1g). Viral antigen was not detected in human CD34 expressing (endothelial) cells, and only a few human vimentin expressing (mesenchymal) cells were positive for viral nucleoprotein (Fig. 1g). To identify the epithelial cell types in the lung tissue that are susceptible to SARSCoV-2 infection, we further identified infected cells by assessing co-localization of viral nucleoprotein with antibodies against acetylated alpha-tubulin IV (ciliated cells), CC10 (club cells), HT1-56 (alveolar type I [AT1] pneumocytes), and pro-SP-C (alveolar type II [AT2] pneumocytes) (Fig. 1h). We were able to clearly identify virus antigen in cells which expressed pro-SP-C or acetylated alpha-tubulin IV; we did not detect virus antigen in HT1-56 or CC10 positive cells (Fig. 1h). These results demonstrate that SARS-CoV-2 has limited tropism in the lung with AT2 pneumocytes and ciliated airway epithelial cells being the predominant lung cells infected by virus.

\section{SARS-CoV-2 pathogenesis in LoM}


To evaluate the cytopathogenic effects of SARS-CoV-2 during acute infection of human lung tissue in LoM, we used a combination of histological analysis and electron microscopy. Histopathologic analysis revealed several features of early diffuse alveolar damage that have been described in lung tissues of COVID-19 patients including the accumulation of proteinaceous exudate and fibrin in alveolar spaces, desquamation of pneumocytes, multi- nucleated cell formation, and the appearance of fibrin thrombi in small vessels (Fig. 2) ${ }^{25-27}$. Proteinaceous exudate, including large globules of protein material, was observed in alveolar spaces, which overlapped with areas of virus accumulation (Fig. 2a,b). As early as 2 days post-infection, desquamation of pneumocytes was also noted; there were a large number of virally infected cells fully detached or detaching from the alveolar basement membrane into the alveolar space (Fig. 2c,d). Infected multi-nucleated cells were also observed (Fig. 2c). While the formation of hyaline membranes was not noted, fibrin was detected in alveolar spaces (Fig. 2e). Importantly, we observed multiple occluded vessels containing fibrin thrombi as reported in the lungs of COVID-19 patients (Fig. $2 f, g)^{25-27}$. Electron microscopy demonstrated the normal architecture and integrity of uninfected AT2 pneumocytes that were present in human lung tissue obtained from LoM two days post-infection (Fig. $2 \mathrm{~h})$. In contrast, AT2 cells containing virus particles in the same sample had swollen mitochondria with loss of matrix and cristae as well as rough endoplasmic reticula with distended cisternae, protein accumulation, and virus particles (Fig. 2i). Degenerative SARS-CoV-2 infected AT2 cells detached from the alveolar basal membrane could also be observed in the alveolar luminal space (Fig. 2j). Higher magnification revealed subcellular accumulation of virus containing vesicles indicative of virus replication and egress. Virions with electron dense nucleocapsids and distinctive crown-like spikes were observed (Fig. 2i,j). Consistent with previous reports in human airway epithelial cell cultures and portmortem lung samples ${ }^{26,28}$, virions produced by human lung cells were pleomorphic in size (69 to 112 $\mathrm{nm}$ ) and shape. Despite the extensive damage inflicted in the lung tissue by the virus, the endothelium in the majority of blood vessels was intact with tight junctions, numerous pinocytotic vesicles, and normal mitochondria and endoplasmic reticulum (Fig. 2k,l). Virions were not detected within endothelial cells in agreement with a lack of infection as per our immunofluorescence analysis (Fig. 1g and Fig. 2k,l). However, pleomorphic virions were present in capillary lumen surrounded by fibrillar protein deposits and cell debris (Fig. 2k,l). Together, these results demonstrate that acute SARS-CoV-2 infection of LoM closely resembles infection of human lung in humans and is highly cytopathic resulting in significant injury to the fragile alveolar lung structures.

To determine the effect of SARS-CoV-2 infection on human gene transcription, we performed RNAsequencing analysis of human lung tissues collected from animals 2, 6 and 14 days post- infection. Abundant viral transcripts were detected in infected lung tissue, ranging from $0.55 \%$ to $3.6 \%$ of the total reads at 2 days post-infection (Extended Data Table 3). Viral transcripts were still abundant but lower at 6 days and 14 days post-infection (Extended Data 3). Sequencing data was consistent with previously identified canonical SARS-CoV-2 transcripts $^{29}$ and confirmed maintenance of the furin cleavage site throughout the course of infection in vivo. Analysis of human gene transcripts revealed 1,504 differentially expressed cellular genes between naïve and infected human lung tissue at 2 days postexposure, the peak of infection (Fig. 3a, Supplementary Tables 1 and 2) (adjusted p value $<0.05$ after 
correcting for multiple testing). Of these, 1,043 genes were up-regulated and 461 genes were down regulated in the infected human lung tissue relative to non-infected lung tissue (Fig. 3a, Supplementary Tables 1 and 2). Notably, numerous interferon-stimulated genes (ISGs) and inflammatory cytokine genes, including pro-inflammatory cytokines genes IL6, CXCL8 (IL-8), CXCL10 (IP-10), TNF, and CCL5 (RANTES) were potently induced in infected lung tissue (Supplementary Tables 1 and 2). We also observed dramatic upregulation of IFNB1 expression ( $>1,000$ fold) at 2 days post- exposure, suggesting that this cytokine plays a key role in the antiviral response to SARS-CoV-2 (Supplementary Tables 1 and 2). Gene set enrichment analysis (GSEA) showed over 840 gene pathways significantly upregulated $(p<0.05)$ including response to type 1 interferon $(p=0.0011)$, response to virus $(p=0.0010)$, innate immune response $(p=0.0010)$, cytokine mediated signaling $(p=0.0010)$, cytokine production $(p=0.0010)$, response to stress $(p=0.0010)$, inflammatory response, $(p=0.0010)$, NIK NF-KB signaling $(p=0.0011)$, acute inflammatory response $(p=0.0035)$, regulation of cell death $(p=0.0030)$, and coagulation pathways $(p=0.0453)$ (Fig. $3 b)$. Complement activation, which contributes to SARS-CoV pathogenesis in mouse models ${ }^{14}$, was also increased ( $p=0.0470)$ (Fig. 3b). Importantly, analysis of host gene expression at later time points demonstrated a sustained upregulation of antiviral and inflammatory genes that in some instances (e.g. ISG15, IFITM1, TNF, CXCL9) persisted for up to 14 days post-infection (last time analyzed) (Fig. 3c,d, Extended Data Table 4, Supplementary Tables 1 and 2). These results demonstrate that acute SARS-CoV2 infection causes a potent and sustained upregulation of innate immune responses in virus-infected human lung tissue.

\section{EIDD-2801 pre-exposure prophylaxis}

Currently, there is no vaccine to prevent SARS-CoV-2 infection or effective therapy to treat patients with COVID-19. The ribonucleoside analog $\beta$-D-N $N^{4}$-hydroxycytidine $(\mathrm{NHC})$ has been shown to broadly inhibit coronavirus infection in vitro in human airway epithelial cell cultures, with potent activity against SARSCoV-2 as well as SARS-CoV, MERS-CoV, and bat SARS- like and MERS-like coronaviruses ${ }^{7}$. We therefore tested the ability of prophylactic EIDD-2801 (also known as MK-4482), the oral pro-drug of NHC, to inhibit SARS-CoV-2 replication in vivo. For this purpose, LoM were administered EIDD-2801 starting $12 \mathrm{~h}$ prior to SARS-CoV-2 exposure and every $12 \mathrm{~h}$ thereafter (Fig. 4a, Extended Data Table 5). Our results show that EIDD-2801 had a dramatic effect on virus infection, significantly reducing the number of infectious particles in the human lung tissue of EIDD-2801 treated animals in two independent experiments (Fig. 4b,c) by over 100,000 fold (Fig. 4c,d). Furthermore, in contrast to EIDD-2801 treated mice, abundant cell debris and nucleoprotein positive cells could be readily observed in the alveolar lumen of vehicle control treated mice consistent with the extensive pathogenic effects inflicted on the lung by SARS-CoV-2 (Fig.4e,f). These results demonstrate that prophylactic administration EIDD-2801 is highly effective at preventing SARS-CoV-2 infection and pathogenesis in vivo.

\section{Discussion}


The zoonotic transmission of the pathogenic SARS-CoV-2 resulted in a pandemic that has inflicted significant morbidity and mortality as well as dire world-wide economic and social consequences. Herein, we describe a unique model for the in vivo study of human coronavirus infection particularly well suited to model distal human lung virus infection. Our results demonstrate replication of all known recently emerged human coronaviruses in LoM.

Importantly, our results demonstrate that WIV1-CoV and SHC014-CoV, two pre-pandemic endogenous bat viruses can replicate relatively efficiently in LoMs suggesting that coronaviruses circulating in bats have future pandemic potential without the need for further adaptation to the human host. We also show that acute SARS-CoV-2 infection of LoM resulted in significant lung injury and exhibited key features of the extensive pathology observed in lung tissues obtained from patients with severe SARS-CoV-2 infection, including desquamation of alveolar epithelial cells, multi-nucleated cell formation, and fibrin thrombi ${ }^{25,27,30,31}$. In agreement with analyses of bronchoalveolar fluid obtained from COVID-19 patients and post-mortem patient lung samples ${ }^{12,32}$, we also observed a significant increase in multiple ISGs in SARS-CoV-2 infected human lung tissue of LoM. In addition, we observed a robust induction in IFNB1 gene expression in human lung tissue early during acute infection followed by a decline in expression. Interestingly, an analysis of post-mortem COVID-19 patient lungs ${ }^{12}$ did not reveal increased IFNB1 expression and it has been shown in vitro that its expression is blocked during SARS- CoV infection ${ }^{33,34}$. These results suggest that in human lung tissue IFNB1 gene expression is induced during the acute phase of SARS-CoV-2 infection. We also observed increased expression of several human cytokine genes in LoM infected with SARS-CoV-2. A substantial number of these cytokines were also increased in the serum of COVID-19 patients and post- mortem lung tissue samples further establishing the similarities between LoM and human infection with SARS-CoV-2 ${ }^{12,35}$. Currently, there is no vaccine to prevent or therapeutics to treat COVID-19. Pre-exposure prophylaxis approaches to infectious diseases have proven to be highly efficacious and can contribute to reduce the risk of infection. The continued global spread of the virus and its associated morbidity and mortality are strong incentives to the development of prevention strategies for COVID-19. In this regard, NHC was shown to have broad activity against human and bat coronavirus infection in vitro ${ }^{7}$. In addition, prophylactic and therapeutic administration of its oral pro-drug EIDD-2801 reduced SARS-CoV and MERS-CoV replication and pathogenesis in mice ${ }^{7}$. Here we show that prophylactic administration of EIDD-2801 efficiently prevents SARS-CoV-2 infection in vivo highlighting its potential utility as an effective prophylactic agent against SARS-CoV-2 and other past and future zoonotic coronaviruses. There are some limitations of our study including the fact that LoM do not possess the human nasal airway structures that are thought to be early sites of SARS-CoV-2 replication in humans ${ }^{36}$. Since LoM do not have an autologous human adaptive immune system they reflect the direct effect of viruses on their targets and bystander cells as well as their innate immune response to infection. Collectively, our results indicate that LoM reflect the pathogenic effects inflicted by SARS-CoV-2 on the human lung and demonstrate their utility as a single in vivo platform to evaluate and compare the replication and pathogenesis of past, present, and future pre-emergent, epidemic, and pandemic coronaviruses accelerating the development and testing of pre-exposure prophylaxis agents such as EIDD-2801. 


\section{References}

1. Sheahan, T. P. et al. An orally bioavailable broad-spectrum antiviral inhibits SARS-CoV- 2 in human airway epithelial cell cultures and multiple coronaviruses in Sci Trans/ Med 12, doi:10.1126/scitranslmed.abb5883 (2020).

2. Jiang, R. et al. Pathogenesis of SARS-CoV-2 in Transgenic Mice Expressing Human AngiotensinConverting Enzyme 2. Cell, doi:10.1016/j.cell.2020.05.027 (2020).

3. Menachery, V. D. et al. A SARS-like cluster of circulating bat coronaviruses shows potential for human emergence. Nat Med 21, 1508-1513, doi:10.1038/nm.3985 (2015).

4. Menachery, V. D. et al. SARS-like WIV1-CoV poised for human emergence. Proc Natl Acad Sci U S A 113, 3048-3053, doi:10.1073/pnas.1517719113 (2016).

5. Boni, M. F. et al. Evolutionary origins of the SARS-CoV-2 sarbecovirus lineage responsible for the COVID-19 pandemic. Nat Microbiol, doi:10.1038/s41564-020-0771-4 (2020).

6. Cui, J., Li, \& Shi, Z. L. Origin and evolution of pathogenic coronaviruses. Nat Rev Microbio/ 17, 181192, doi:10.1038/s41579-018-0118-9 (2019).

7. Dong, E., Du, H. \& Gardner, L. An interactive web-based dashboard to track COVID-19 in real time. Lancet Infect Dis 20, 533-534, doi:10.1016/S1473-3099(20)30120-1 (2020).

8. Wahl, et al. Precision mouse models with expanded tropism for human pathogens. Nat Biotechnol 37, 1163-1173, doi:10.1038/s41587-019-0225-9 (2019).

9. Franks, T. J. et al. Resident cellular components of the human lung: current knowledge and goals for research on cell phenotyping and function. Proc Am Thorac Soc 5, 763-766, doi:10.1513/pats.200803-025HR (2008).

10. Dinnon, H., 3rd et al. A mouse-adapted model of SARS-CoV-2 to test COVID-19 countermeasures. Nature, doi:10.1038/s41586-020-2708-8 (2020).

11. Gralinski, L. E. et al. Complement Activation Contributes to Severe Acute Respiratory Syndrome Coronavirus Pathogenesis. mBio 9, doi:10.1128/mBio.01753-18 (2018).

12. Rockx, B. et al. Comparative pathogenesis of COVID-19, MERS, and SARS in a nonhuman primate Science 368, 1012-1015, doi:10.1126/science.abb7314 (2020).

13. Blanco-Melo, et al. Imbalanced Host Response to SARS-CoV-2 Drives Development of COVID-19. Cell 181, 1036-1045 e1039, doi:10.1016/j.cell.2020.04.026 (2020).

14. McCray, P. B., Jr. et al. Lethal infection of K18-hACE2 mice infected with severe acute respiratory syndrome coronavirus. J Viro/ 81, 813-821, doi:10.1128/JVI.02012-06 (2007).

15. Bao, L. et al. The pathogenicity of SARS-CoV-2 in hACE2 transgenic mice. Nature, doi:10.1038/s41586-020-2312-y (2020).

16. Cockrell, S. et al. A mouse model for MERS coronavirus-induced acute respiratory distress syndrome. Nat Microbio/ 2, 16226, doi:10.1038/nmicrobiol.2016.226 (2016). 
17. Menachery, V. D. et al. Middle East Respiratory Syndrome Coronavirus Nonstructural Protein 16 Is Necessary for Interferon Resistance and Viral Pathogenesis. mSphere 2, doi:10.1128/mSphere.00346-17 (2017).

18. Song, Z. et al. From SARS to MERS, Thrusting Coronaviruses into the Spotlight. Viruses 11, doi:10.3390/v11010059 (2019).

19. Yan, R. et al. Structural basis for the recognition of SARS-CoV-2 by full-length human ACE2. Science 367, 1444-1448, doi:10.1126/science.abb2762 (2020).

20. Walls, C. et al. Structure, Function, and Antigenicity of the SARS-CoV-2 Spike Glycoprotein. Cell 181, 281-292 e286, doi:10.1016/j.cell.2020.02.058 (2020).

21. Hoffmann, M. et al. SARS-CoV-2 Cell Entry Depends on ACE2 and TMPRSS2 and Is Blocked by a Clinically Proven Protease Inhibitor. Cell 181, 271-280 e278, doi:10.1016/j.cell.2020.02.052 (2020).

22. Raj, S. et al. Dipeptidyl peptidase 4 is a functional receptor for the emerging human coronavirusEMC. Nature 495, 251-254, doi:10.1038/nature12005 (2013).

23. Li, W. et al. Angiotensin-converting enzyme 2 is a functional receptor for the SARS coronavirus. Nature 426, 450-454, doi:10.1038/nature02145 (2003).

24. Menter, T. et al. Post-mortem examination of COVID19 patients reveals diffuse alveolar damage with severe capillary congestion and variegated findings of lungs and other organs suggesting vascular dysfunction. Histopathology, doi:10.1111/his.14134 (2020).

25. Carsana, L. et al. Pulmonary post-mortem findings in a series of COVID-19 cases from northern Italy: a two-centre descriptive study. Lancet Infect Dis, doi:10.1016/S1473-3099(20)30434-5 (2020).

26. Meyerholz, D. K., Lambertz, M. \& McCray, P. B., Jr. Dipeptidyl Peptidase 4 Distribution in the Human Respiratory Tract: Implications for the Middle East Respiratory Syndrome. Am J Patho/ 186, 78-86, doi:10.1016/j.ajpath.2015.09.014 (2016).

27. Siu, L. et al. Severe acute respiratory syndrome coronavirus M protein inhibits type I interferon production by impeding the formation of TRAF3.TANK.TBK1/IKKepsilon complex. J Biol Chem 284, 16202-16209, doi:10.1074/jbc.M109.008227 (2009).

28. Spiegel, M. et al. Inhibition of Beta interferon induction by severe acute respiratory syndrome coronavirus suggests a two-step model for activation of interferon regulatory factor 3 . J Virol 79, 2079-2086, doi:10.1128/JVI.79.4.2079-2086.2005 (2005).

29. Zhou, Z. et al. Heightened Innate Immune Responses in the Respiratory Tract of COVID- 19 Patients. Cell Host Microbe 27, 883-890 e882, doi:10.1016/j.chom.2020.04.017 (2020).

30. Zhang, H. et al. Histopathologic Changes and SARS-CoV-2 Immunostaining in the Lung of a Patient With COVID-19. Ann Intern Med 172, 629-632, doi:10.7326/M20-0533 (2020).

31. Xu, Z. et al. Pathological findings of COVID-19 associated with acute respiratory distress syndrome. Lancet Respir Med 8, 420-422, doi:10.1016/S2213-2600(20)30076-X (2020).

32. Kim, et al. The Architecture of SARS-CoV-2 Transcriptome. Cell 181, 914-921 e910, doi:10.1016/j.cell.2020.04.011 (2020). 
33. Zhu, N. et al. A Novel Coronavirus from Patients with Pneumonia in China, 2019. N Engl J Med 382, 727-733, doi:10.1056/NEJMoa2001017 (2020).

34. Tian, S. et al. Pulmonary Pathology of Early-Phase 2019 Novel Coronavirus (COVID- 19) Pneumonia in Two Patients With Lung Cancer. J Thorac Oncol 15, 700-704, doi:10.1016/j.jtho.2020.02.010 (2020).

35. Qin, C. et al. Dysregulation of immune response in patients with COVID-19 in Wuhan, Clin Infect Dis, doi:10.1093/cid/ciaa248 (2020).

36. Hou, Y. J. et al. SARS-CoV-2 Reverse Genetics Reveals a Variable Infection Gradient in the Respiratory Tract. Cell, doi:10.1016/j.cell.2020.05.042 (2020).

\section{Methods}

\section{Experimental design}

Human lung-only mice (LOM) were used as an in vivo model to evaluate infection of lung tissue with recombinant coronaviruses SARS-CoV, MERS-CoV, and SARS-CoV-2 as well as full length bat coronaviruses WIV1 and SHC014 4,5,37,38. Viruses were directly injected into the human lung tissue of LoMs and lung tissue collected either 2, 6, or 14 days post-exposure for virus titer determination and/or analysis by histology, electron microscopy, or RNA-seq.

\section{Construction of humanized mice}

LoMs were constructed with 1-2 human lung implants by surgically implanting human lung tissue (Advanced Bioscience Resources) subcutaneously into the upper and lower back of male and female 1221 week old NOD.Cg-Prkdc ${ }^{\text {scid }} \| 2 \mathrm{rg}^{\mathrm{tm} 1 \text { Wjl }} / \mathrm{SzJ}$ mice [NSG mice; The Jackson Laboratory] as previously described ${ }^{17}$. Engraftment of lung tissue was assessed by palpation and by 8 weeks post-surgery animals were ready for experimentation. Mice were housed and maintained by the Division of Comparative Medicine at the University of North Carolina-Chapel Hill and in accordance to the NIH Guide for the Care and Use of Laboratory Animals.

\section{Production of coronavirus stocks and infection of humanized mice}

Stocks of wild-type SARS-CoV, MERS-CoV (HCoV-EMC/2012), SHC014-CoV, and WIV1- CoV were derived from infectious virus clones and were prepared and titered on Vero E6 (SARS-CoV, SHC014-CoV, and WIV1-CoV) or Vero CCL81 cells (MERS-CoV) (American Type Culture Collection) as previously described ${ }^{4,5,9,17}$. A clinical isolate of SARS-CoV-2 (2019-nCoV/USA-WA1/2020) was obtained from the U.S. Centers for Disease Control and Prevention (GenBank accession no. MN985325.1) and passaged 
twice in Vero E6 cells to create a passage 5 working stock ${ }^{36}$. For the infection of mice with coronavirus, the fur over the human lung tissue of anesthetized mice was shaved and virus (10 5 PFU in 100 ul PBS or $3 \times 10^{5}$ PFU in 100 ul PBS for bat coronavirus inoculations) was injected directly into the lung tissue. To evaluate the in vivo inhibitory activity of EIDD-2801, mice were administered $500 \mathrm{mg} / \mathrm{kg}$ EIDD-2801 or vehicle control (10\% PEG and 2.5\% Cremophor RH40 in water) via oral gavage starting $12 \mathrm{~h}$ prior to SARSCoV-2 exposure and every $12 \mathrm{~h}$ thereafter. At necropsy, human lung tissues were collected, weighed, homogenized, and stored at $-80^{\circ} \mathrm{C}$ until tittering on Vero E6 cells. Titers below the limit of the assay (50 $\mathrm{PFU} / \mathrm{mL}$ ) were assigned a value of $25 \mathrm{PFU} /$ gram.

\section{H\&E staining of human lung tissue}

Human lung tissues collected from LoM were fixed in $10 \%$ formalin, paraffin embedded, and cut into $5 \mu \mathrm{m}$ sections which were mounted onto Superfrost Plus slides (Fisher Scientific). Tissue sections were incubated at $60^{\circ} \mathrm{C}$ for $1 \mathrm{~h}$, deparaffinized with xylene $(2 \times 3 \mathrm{~min})$ and graded ethanol $(100 \% 2 \times 3 \mathrm{~min}$, $95 \% 1 \times 3 \mathrm{~min}, 80 \% 1 \times 3 \mathrm{~min}, 70 \% 1 \times 3 \mathrm{~min}$ ), and stained with hematoxylin followed by eosin. Tissue sections were then mounted and imaged on a Nikon Eclipse Ci microscope using Nikon Elements BR software (version 4.30.01) with a Nikon Digital Sight DS-Fi2 camera. Brightness, contrast and white balance were adjusted on whole images in Adobe Photoshop (CS6).

\section{Immunohistochemical analysis of coronavirus infection}

Immunohistochemistry was performed as previously described ${ }^{17}$. Briefly, fixed (10\% formalin) human lung tissues collected from coronavirus-infected LoM were paraffin embedded and sectioned (5 um). Tissue sections mounted on Superfrost Plus slides (Fisher Scientific) were deparaffinized as described above. Following antigen retrieval using Diva Decloaker (BioCare Medical), non-specific binding was blocked using Background Sniper (BioCare Medical). Tissue sections were then incubated with primary antibodies against SARS-CoV or MERS-CoV nucleocapsid overnight at $4^{\circ} \mathrm{C}$. Tissue sections incubated with rabbit lgG were used as isotype controls. Tissue sections were then washed in TBST and the endogenous peroxidase activity blocked with hydrogen peroxide. Tissue sections were developed using the MACH-3 polymer system (BioCare Medical) and 3,3'-diaminobenzidine (DAB) (Vector Laboratories), counterstained with hematoxylin, and mounted. Tissue sections were imaged on a Nikon Eclipse Ci microscope using Nikon Elements BR software (version 4.30.01) with a Nikon Digital Sight DS-Fi2 camera. Adobe Photoshop (CS6) was used to adjust brightness, contrast and white balance on whole images.

\section{Immunofluorescence analysis of SARS-CoV2 infection}

Human lung tissues collected from mice were fixed in 10\% formalin and paraffin embedded. Immunofluoresence staining of 5 um tissue sections was performed as previously described ${ }^{17}$. Briefly, 
following deparaffinization and antigen retrieval (Diva Decloaker), tissue sections were incubated with a $10 \%$ normal donkey serum solution with $0.1 \%$ Triton X-100 in $1 \times$ PBS to block non-specific binding.

Tissue sections were then incubated overnight with primary antibodies at $4^{\circ} \mathrm{C}$ followed by incubation with fluorescent conjugated secondary antibodies (Supplementary Table 7). Primary antibodies were directed against SARS nucleoprotein and human cytokeratin 19, CD34, vimentin, acetylated alpha-tubulin IV, CC10, HT1-56, and pro-SP-C (Supplementary Table 7). Background autofluorescence was then quenched using a $0.1 \%$ Sudan Black B solution in $80 \%$ ethanol prior to staining with DAPI. Slides were mounted and then imaged using an Olympus BX61 upright wide-field microscope using Volocity software (version 6.3) with a Hamamatsu ORCA RC camera. Appropriate negative controls without primary antibodies were also imaged using the same exposure time as matching stained sections. Whole image contrast, brightness, and pseudocoloring were adjusted using ImageJ/Fiji (Version 2.0.0-rc-69/1.51w) and Adobe Photoshop (version CS6).

\section{RNA in situ hybridization (RNA-ISH) analysis of SARS-CoV2 infection}

RNA-ISH was performed on $10 \%$ formalin fixed, paraffin-embedded, $5 \mu \mathrm{m}$ sections of human lung tissues using the RNAscope 2.5 HD Reagent Kit according to the manufacturer's instructions (Advanced Cell Diagnostics). Briefly, tissue sections were mounted on Superfrost Plus microscope slides (Fisher Scientific), heated at $60^{\circ} \mathrm{C}$ for $1 \mathrm{~h}$, deparaffinized in xylene $(2 \times 5$ minutes $)$ and $100 \%$ ethanol $(2 \times 2$ minutes), and air-dried. Tissue sections were then incubated with hydrogen peroxide to block endogenous peroxidases for 10 min at RT, followed by epitope retrieval (Advanced Cell Diagnostics) for $30 \mathrm{~min}$ in a $95^{\circ} \mathrm{C}$ water bath. Subsequently, tissue sections were immediately washed in double distilled water then dehydrated in $100 \%$ ethanol for 2 min before air-drying. Tissue sections were then incubated with Protease Plus (Advanced Cell Diagnostics) for $30 \mathrm{~min}$ at $40^{\circ} \mathrm{C}$ in a HybEZ hybridization oven (Advanced Cell Diagnostics). Sections were rinsed 3 times in double distilled water and then incubated with prewarmed target probe designed to hybridize with the spike protein of SARS-CoV-2 (Cat. Number 848561, Advanced Cell Diagnostics) for $2 \mathrm{~h}$ at $40^{\circ} \mathrm{C}$. Tissue sections were then washed and the signal amplified according to the manufacturer's instructions and developed using alkaline phosphatase and Fast Red substrate. Tissue sections were counterstained with DAPI, mounted with Prolong® Gold (Invitrogen), and imaged on an EVOS M5000 microscope (Invitrogen).

\section{Electron microscopy analysis of SARS-CoV2 infection}

Small pieces of human lung tissue collected from SARS-CoV-2 infected LoM at two days post- infection were fixed in $4 \%$ paraformaldehyde/ $2.5 \%$ glutaraldehyde in $0.15 \mathrm{M}$ sodium phosphate buffer, $\mathrm{pH} 7.4$, for 2 $\mathrm{h}$ at RT. The tissues were subsequently transferred to $10 \%$ formalin for 7 days. Specimens were washed in $0.1 \mathrm{M}$ sodium cacodylate, $\mathrm{pH} 7.4$, then post-fixed with $1 \%$ cacodylate-buffered osmium tetroxide for 1 h. After washing in $0.05 \mathrm{M}$ sodium cacodylate buffer, $\mathrm{pH} 7.0$, the samples were treated with $1 \%$ tannic 
acid in $0.05 \mathrm{M}$ sodium cacodylate buffer for $30 \mathrm{~min}$ to enhance tissue contrast and preserve structure ${ }^{39}$. Tissue pieces were washed in deionized water, dehydrated in ethanol, and placed through two exchanges of propylene oxide before infiltration and embedment in PolyBed 812 epoxy resin (Polysciences). Semithin $(1 \mu \mathrm{m})$ sections of tissue blocks were cut and stained with $1 \%$ toluidine blue in $1 \%$ sodium borate for examination by light microscopy. Ultra-thin $(70 \mathrm{~nm})$ sections were cut of selected regions of interest, mounted on 200 mesh copper grids and stained with $4 \%$ aqueous uranyl acetate and Reynolds' lead citrate. Grids were observed on a JEOL JEM 1230 transmission electron microscope operating at 80kV (JEOL USA, Inc.) and images were acquired with a Gatan Orius SC1000 CCD Digital Camera and Gatan Microscopy Suite software (version 3.0, Gatan, Inc.). Virus particle sizes were measured in Fiji/Image J (version 2.0.0-rc-69/1.52p).

\section{Processing of human lung tissues for RNA-sequencing analysis}

Human lung tissues were collected in RNAlater and kept at $4^{\circ} \mathrm{C}$ for $24 \mathrm{~h}$ prior to storage at $-80^{\circ} \mathrm{C}$ until further processing. To isolate RNA, samples stored in RNAlater were thawed and the tissue transferred to a new tube containing $1 \mathrm{~mm}$ glass beads and $1 \mathrm{~mL}$ Trizol. Tissues were subsequently homogenized using a MagNA Lyser (Roche) for $30 \mathrm{sec}$ at 6,000 rpm. In between rounds of homogenization, tissues were incubated on ice for $1 \mathrm{~min}$. Following tissue homogenization, Trizol homogenate was transferred to a new tube and stored at $-80^{\circ} \mathrm{C}$.

\section{RNA-sequencing analysis}

RNA was extracted from lung samples using a Trizol Plus RNA extraction kit (Thermo Fisher), quantified using a Qubit RNA assay kit and checked for quality using a Bioanalyzer RNA600 Nano kit (Agilent). RNA integrity scores were typically 7.0 and greater. 1 ug of RNA was used to construct libraries for sequencing using a NEBNext Ultra II library prep kit with polyA RNA selection. Barcoded libraries were sequenced on a Novaseq 6,000 2x100 bp following manufacturer's instructions (Illumina). Sequence quality was assessed using FASTQC (v). No issues were detected with the data and quality was typical for RNA extracted from fresh frozen material. A small amount of index hopping was detected $(0.09 \%)$ due to the single indices used in the library preparation. Raw reads were mapped to the human, mouse, and SARSCoV-2 reference genomes simultaneously (GRCh38.p13, GRCm38.p6 M25, NC_045512, respectively) using the BBsplit function in BBmap (version 38.86). This step minimized cross mapping of reads among genomes. We then mapped and quantified on a transcript and gene model basis using STAR (version 2.7.5a) and Salmon (version 1.2.1 $)^{40,41}$. Reads mapping to multiple locations were dropped from analysis. On average, in the lung tissue samples from LoM mice, $80 \%$ of the reads mapped to human (standard deviation: $+/-6 \%$ ), 19\% mapped to mouse (standard deviation: $+/-6 \%$ ) and $1 \%$ mapped to the SARS-Cov-2 genome (standard deviation: $+/-1 \%$ ). The percent of virus ranged from $0.05 \%$ to $3.4 \%$ among 
infected mice, with day 2 mice having the most, which is consistent with the infection titers observed. Samples from naïve mice were $95 \%$ or more human data.

\section{Statistical analysis}

RNA-sequencing data was normalized and interrogated for changes in gene expression using DESeq2 package (version 3.1.1) in $\mathrm{R}$ (version 3.6.3) ${ }^{42}$. We focused the analysis on the naïve controls versus LoM for days 2,6 , and 14 post-infection. Wald's tests were performed contrasting each day versus naïve controls. Because mice were sacrificed at each time point, we treated each day independently and not as a time series. $P$-values were adjusted for multiple testing using a False Discovery Rate using the Benjamini \& Hochberg method ${ }^{43}$. Data was analyzed both jointly and within each treatment compared to naïve controls. Differential expression of outliers was assessed and found insignificant in overall effect. Graphs and summary tables were built in R using ggplot; gene set enrichment was performed using GSEA and GO analysis (tidyverse 1.3.0; PCATools 1.2.0; Sqldf 0.4-11; na.tools 0.3.1; ggbiplot 0.55; ggplot2 3.3.1; dplyr 0.8.4). Specific gene sets of interest were then interrogated for patterns of expression across treatment and time using unsupervised clustering of normalized gene expression counts. Gene Ontology (GO) analysis and visualization were performed with GOrilla ${ }^{44}$. Data was uploaded to the NCBI GEO archive (accession: GSE155286). Virus titers between vehicle control and EIDD-2801 treated LoM were compared with a two-tailed Mann- Whitney U test.

\section{Methods References}

37. Scobey, T. et al. Reverse genetics with a full-length infectious cDNA of the Middle East respiratory syndrome coronavirus. Proc Natl Acad Sci U S A 110, 16157-16162, doi:10.1073/pnas.1311542110 (2013).

38. Yount, B. et al. Reverse genetics with a full-length infectious CDNA of severe acute respiratory syndrome coronavirus. Proc Natl Acad Sci U S A 100, 12995-13000, doi:10.1073/pnas.1735582100 (2003).

39. Simionescu, N. \& Simionescu, M. Galloylglucoses of low molecular weight as mordant in electron microscopy. I. Procedure, and evidence for mordanting effect. J Cell Bio/ 70, 608-621, doi:10.1083/jcb.70.3.608 (1976).

40. Dobin, et al. STAR: ultrafast universal RNA-seq aligner. Bioinformatics 29, 15-21, doi:10.1093/bioinformatics/bts635 (2013).

41. Patro, R., Duggal, G., Love, M. I., Irizarry, R. \& Kingsford, C. Salmon provides fast and bias-aware quantification of transcript expression. Nat Methods 14, 417-419, doi:10.1038/nmeth.4197 (2017). 
42. Love, M. I., Huber, \& Anders, S. Moderated estimation of fold change and dispersion for RNA-seq data with DESeq2. Genome Biol 15, 550, doi:10.1186/s13059-014-0550-8 (2014).

43. Benjamini, Y. \& Hochberg, Y. Controlling the False Discovery Rate: A Practical and Powerful Approach to Multiple Testing. 57, 289-300, doi:10.1111/j.2517- 6161.1995.tb02031.x (1995).

44. Eden, E., Navon, R., Steinfeld, I., Lipson, D. \& Yakhini, Z. GOrilla: a tool for discovery and visualization of enriched GO terms in ranked gene lists. BMC Bioinformatics 10, 48, doi:10.1186/1471-2105-10-48 (2009).

\section{Declarations}

Acknowledgements: We thank current and past members of the Garcia laboratory for technical assistance. The authors also thank technicians at the UNC Animal Histopathology and Laboratory Animal Medicine Core and Division of Comparative Medicine. We also thank the UNC School of Medicine Bioinformatics and Analytics Research Collaborative (BARC) for providing technical support and K. Mollan and B. Shook-Sa of the UNC CFAR Biostatistics Core for providing statistical support.

Funding: This work was supported by funding from National Institutes of Health grants R21Al113736 (RJP), U19Al100625 (RSB), R21Al145372 (LEG), R01Al123010 (AW), R01Al111899 (JVG), R01Al140799 (JVG), R01MH108179 (JVG). The Microscopy Services Laboratory, Department of Pathology and Laboratory Medicine, is supported in part by P30 CA016086 Cancer Center Support Grant to the UNC Lineberger Comprehensive Cancer Center.

Author contributions: AW, CEJ, WY, MK, and CD constructed LoM. AW contributed to experimental design, data interpretation, data presentation, and manuscript writing, coordinated the study, and the preparation of the manuscript. LEG performed the virus inoculations, animal necropsies, virus tittering, processing of lung tissues for RNA extraction, and contributed to the experimental design, planning, data analysis, data interpretation, and manuscript writing. CEJ performed immunofluorescence and H\&E analyses, WY performed immunohistochemistry and H\&E analyses, and MK performed the in-situ hybridization analysis of LoM human lung tissues. KHD, AS, SRL, and KG assisted with the in vivo experiments with coronavirus-infected LoMs. VJM in conjunction with KKW performed the electron microscopy analysis. FBA assisted with the pathohistological analysis. HL, HMK, TZ, POG, performed the RNA-sequencing analysis. EPB and CDJ contributed to design of RNA-sequencing experiments. RJP assisted with the immunofluorescence analysis and contributed to experimental design, data interpretation, data presentation, and manuscript writing. RSB conceived and designed experiments and contributed to data interpretation and manuscript writing. JVG conceived, designed and coordinated the study, conceived and designed experiments, and contributed to data interpretation, data presentation, and manuscript preparation.

Competing interests: The authors have no competing interests. 


\section{Additional Information}

Supplementary information is available for this paper at

Correspondence: Correspondence to J. Victor Garcia.

\section{Reporting summary}

Further information on research design is available in the Nature Research Reporting Summary linked to this paper.

Data availability: Gene-expression data are available at the Gene Expression Omnibus (GEO) repository (accession: GSE155286). All other data is available in the manuscript or the supplementary materials.

\section{Figures}



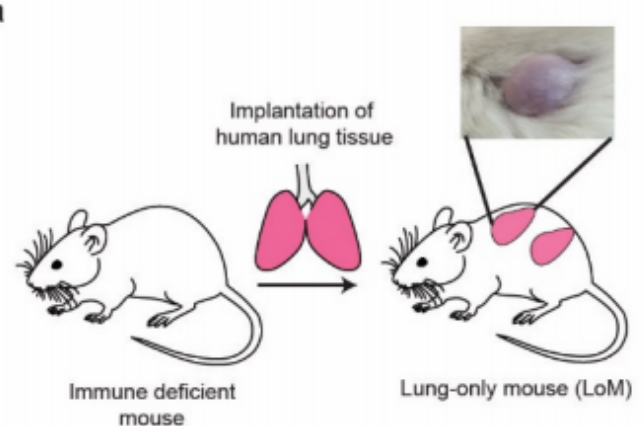

a

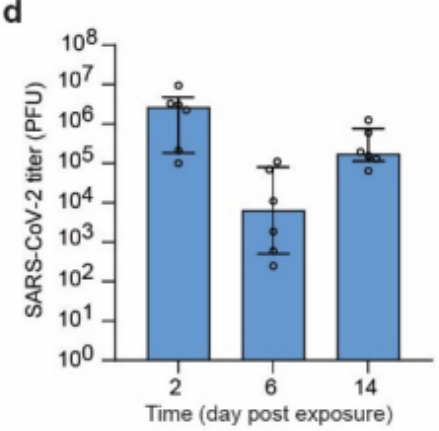

g

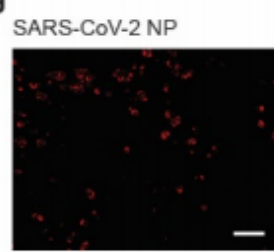

SARS-CoV-2 NP

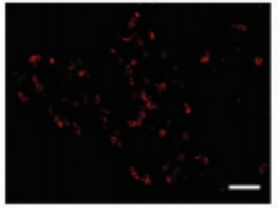

SARS-CoV-2 NP

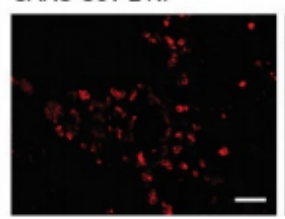

b

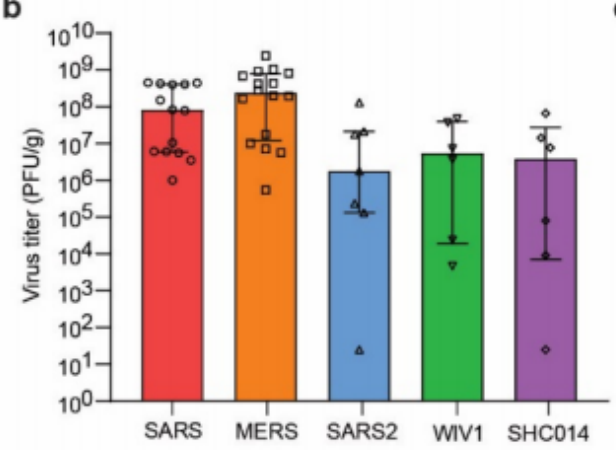

$c_{\text {SARS-COV-2 }}$

exposure

(10 $10^{5}$ PFU/lung)

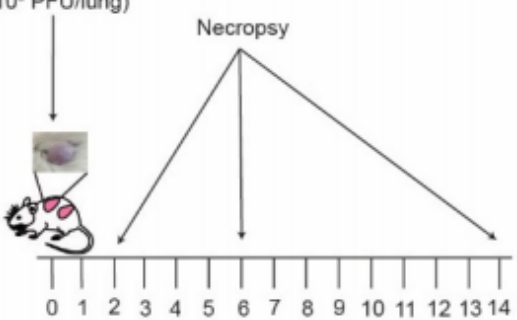

Time (day post SARS-CoV-2 exposure) e

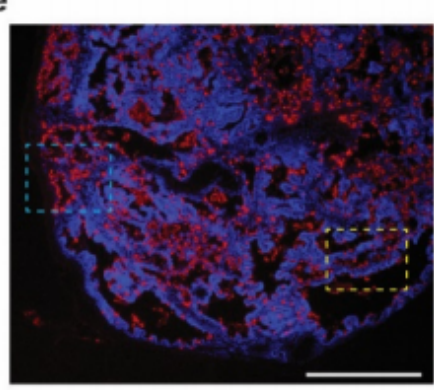

f
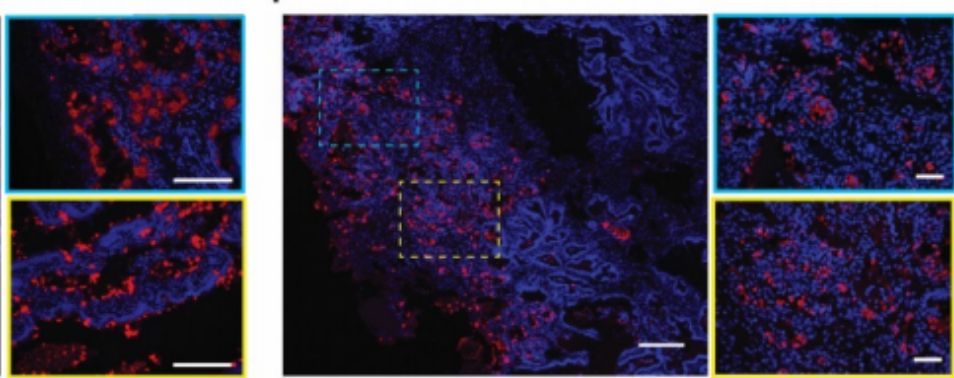

h

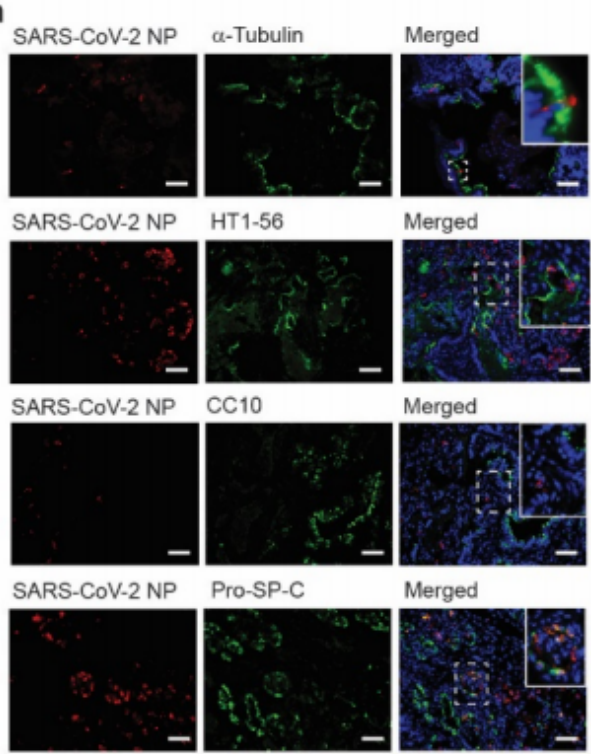

Figure 1

Robust replication of recently emerged human and bat coronaviruses in LoM demonstrate the potential of bat coronaviruses for direct transmission to humans and the predilection of SARS- CoV-2 for infection of human epithelial cells. a, Construction of LoM and image of a human lung implant. b, Titers of virus in the human lung tissue of LoM directly injected with SARS-CoV ( $n=14$, red), MERS-CoV ( $n=16$, orange), SARS-CoV-2 ( $n=7$, blue), WIV1-CoV ( $n=6$, green), or SHC014 $(n=6$, purple) as determined by plaque assay (PFU, plaque forming units). c, SARS-CoV-2 was directly injected into the human lung tissue of LoM. Human lungs were collected at days 2,6 , and 14 post-infection. $d$, SARS-CoV-2 titers in the human lung tissue of LoM 
a

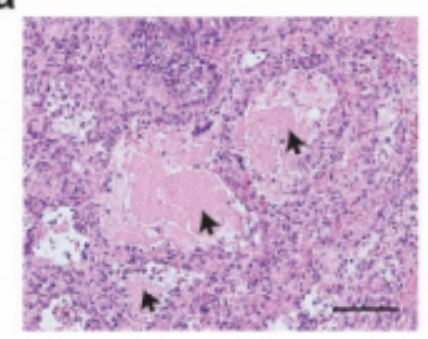

e

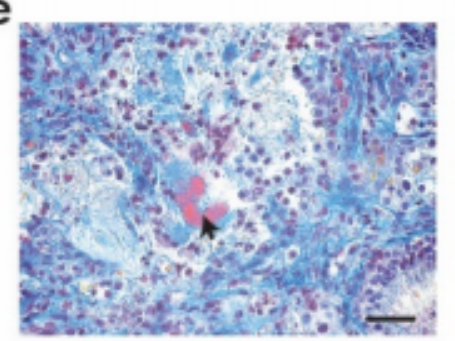

h

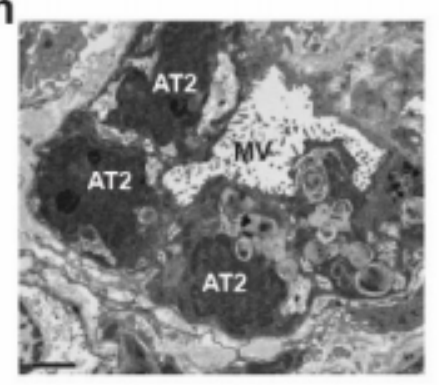

i b

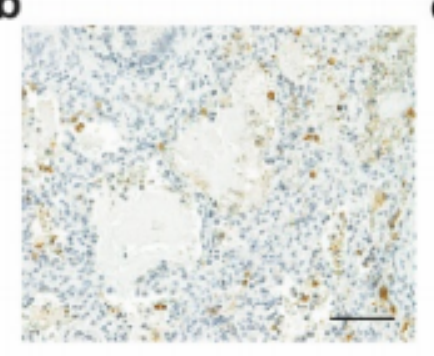

f

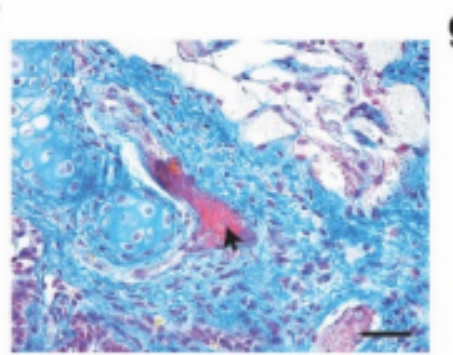

c

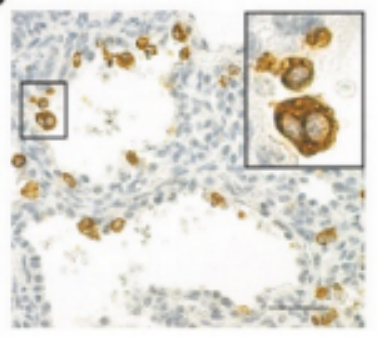

d

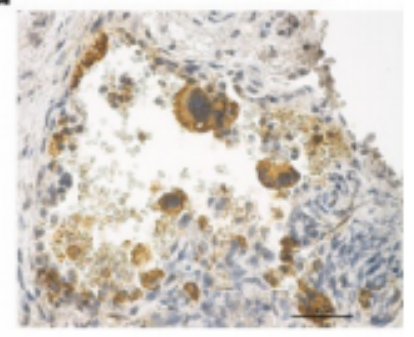

g
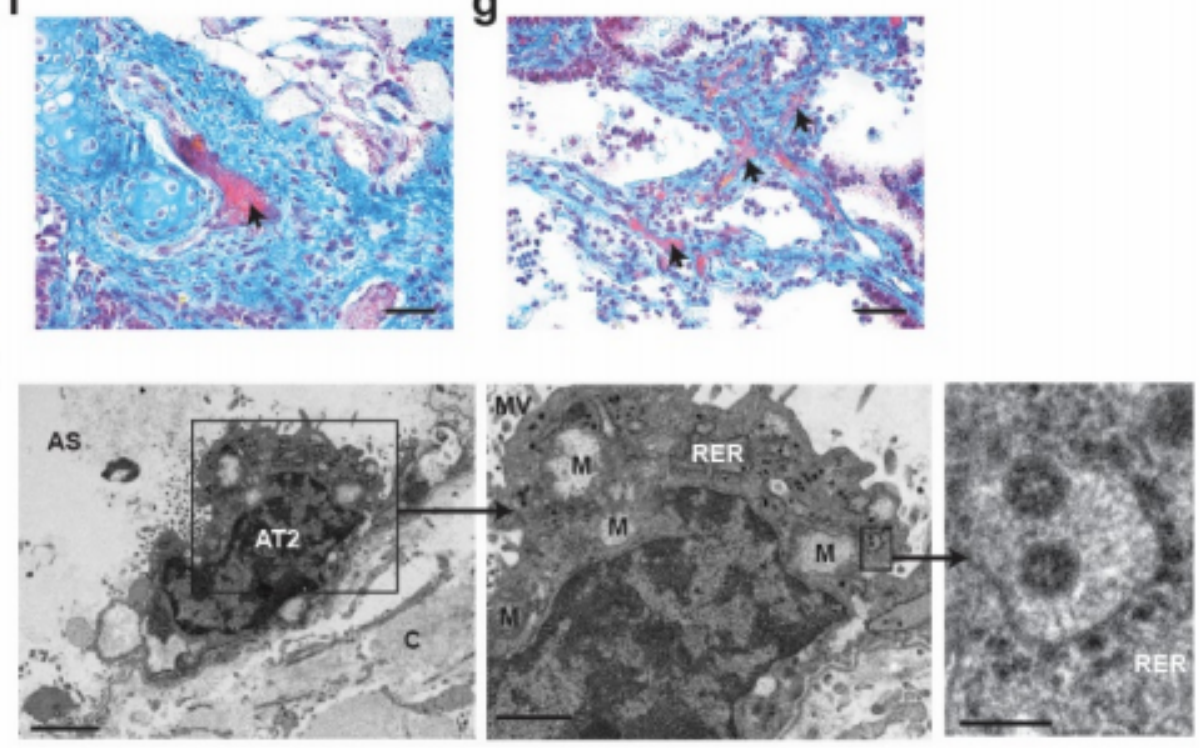

j

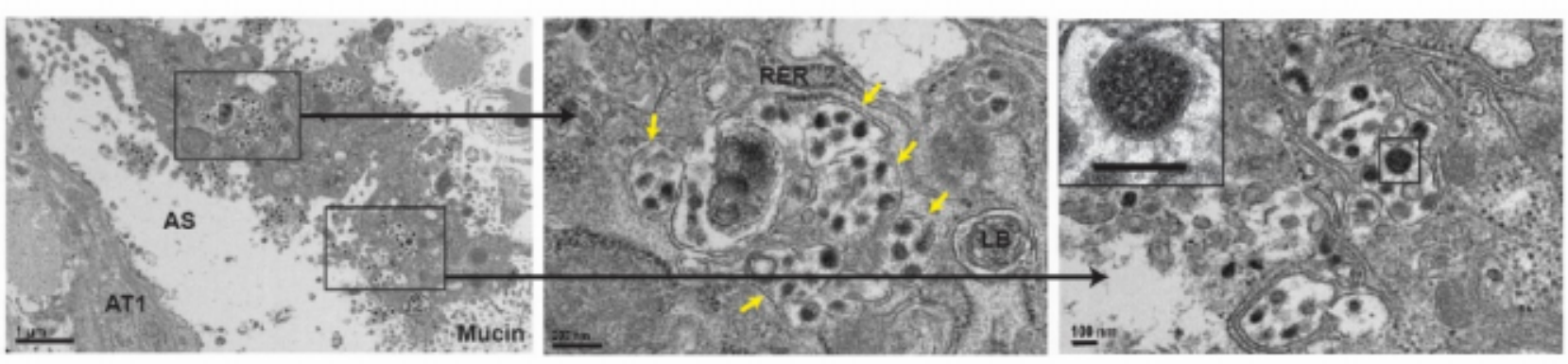

k
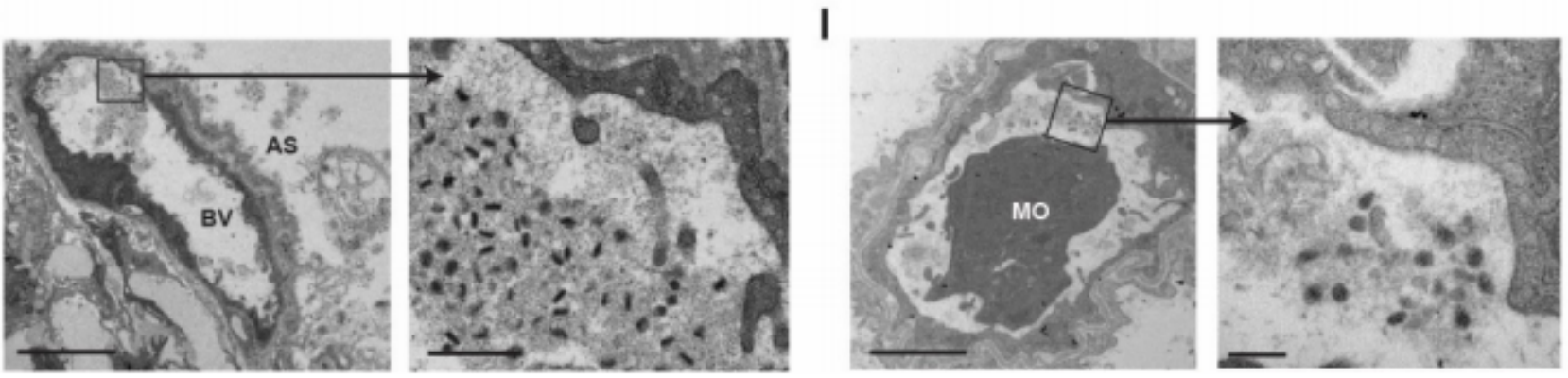

Figure 2

During acute infection, SARS-CoV-2 is highly cytopathic and causes extensive damage to human lung structures. a, H\&E staining of a SARS-CoV-2 infected LoM human lung tissue 2 days post-exposure (scale bar, 100 um, $n=6$ analyzed). Globules of protein material are indicated with arrows. b-d, Immunohistochemical staining for virus nucleoprotein in LoM human lung tissue 2 days following SARSCoV-2 exposure (positive cells, brown; b scale bars, 100 um; c and d scale bars, 50 um, $n=6$ analyzed). e-g, 
Martius Scarlet Blue staining of a SARS-CoV-2 infected LoM human lung tissue 2 days post-exposure (scale bars, 50 um; fibrin, red; collagen, blue, $n=6$ analyzed). Arrows indicate the presence of fibrin (red) in e, alveoli or in $\mathrm{f}$ and $\mathrm{g}$, thrombi of occluded vessels. h-I, Electron microscopy analysis of SARS-CoV-2 infected human lung tissue two days post-exposure ( $n=3$ analyzed). $h$, Uninfected AT 2 cells in an alveolus-like structure. Scale bars, 2 um. i, SARS-CoV-2 infected AT2 cell. Higher magnification images of areas indicated with black boxes show virus particles with dense nucleocapsids in RER. Scale bars, 2 um (left image), 1 um (middle image), and $200 \mathrm{~nm}$ (right image). j, A degenerative SARS-CoV-2 infected cell in the alveolar space. Vesicles filled with virus particles in the middle image are indicated with arrows. Scale bars, 1 um (left image), $200 \mathrm{~nm}$ (middle image), and $100 \mathrm{~nm}$ (right image). $\mathrm{k}$ and I, Blood vessels containing virions, fibrillar protein and cell debris. In k, scale bars, 5 um (left image) and $500 \mathrm{~nm}$ (right image). In I, scale bars, 2 um (right image) and $200 \mathrm{~nm}$ (left image). AS, alveolar space; AT1, alveolar type 1 cells; AT2, alveolar type 2 cells; BV, blood vessel; C, collagen; LB, lamellar body; M, mitochondria; MO, monocyte; MV, microvilli; RER, rough endoplasmic reticulum. In C, i-I, black boxes indicate areas of higher magnification images.

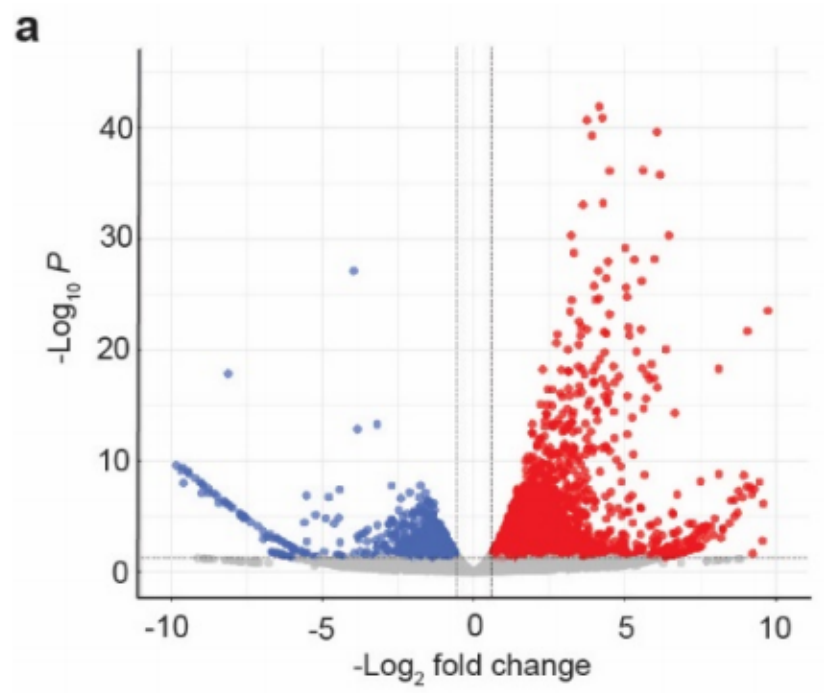

b
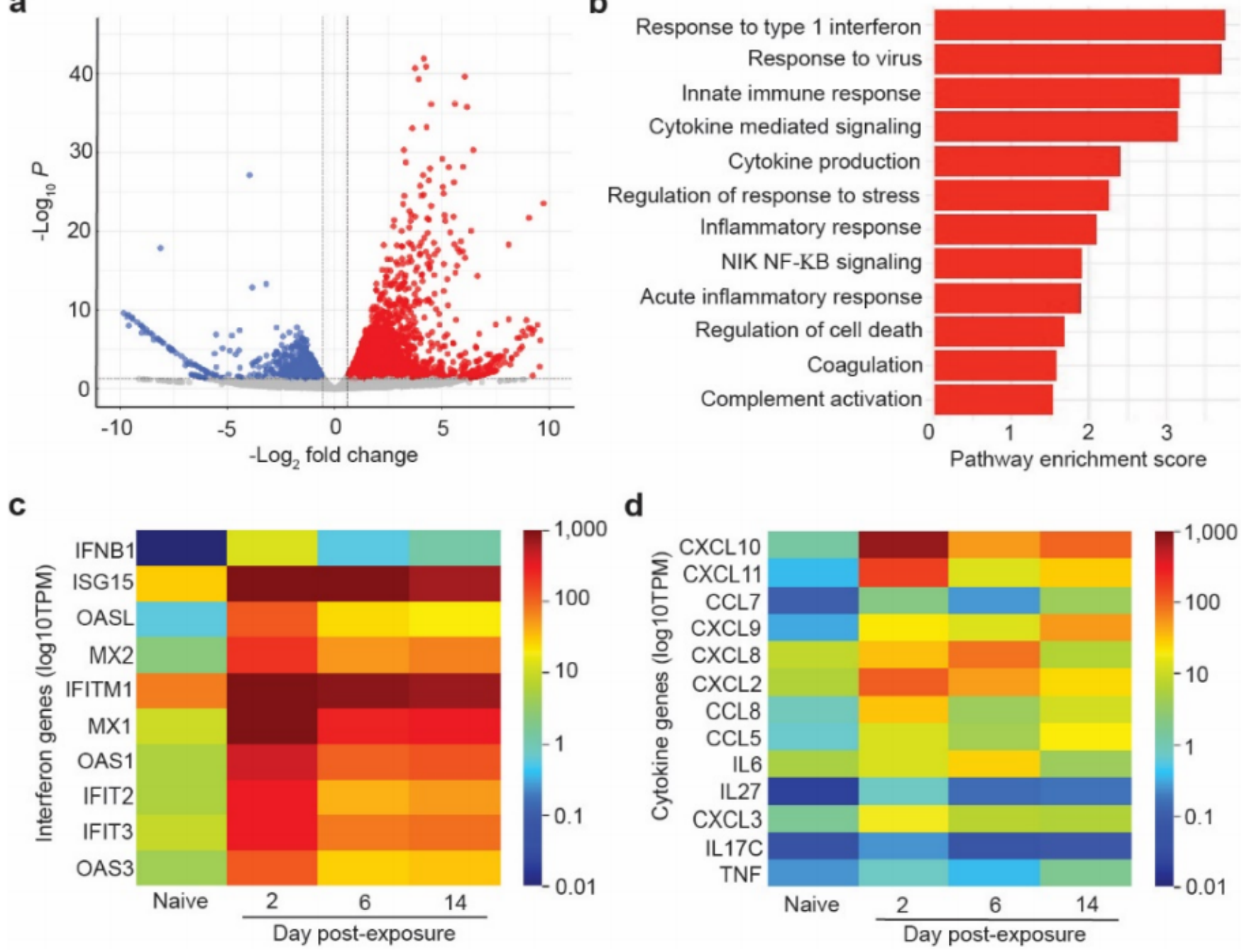

Figure 3 
SARS-CoV-2 infection induces a strong and sustained host innate immune response in human lung tissue. a-d, RNA-sequencing analysis of human lung tissue collected from SARS-CoV-2 infected LoM. a, The plot depicts the up-regulated (red) and down-regulated (blue) genes in SARS-CoV-2 infected human lung tissue two days post-infection ( $n=2)$ compared to human lung tissue from naïve LoM $(n=2)$. The mean - log2-transformed fold change and the multiple testing adjusted - $\log 10 p$ value are shown on the $x$ axis and $y$-axis respectively. Dashed lines show the thresholds of log2-transformed fold change of 1.5 and adjusted $p<0.05$. $b$, Gene set enrichment analysis (GSEA) identified gene sets enriched in SARS-CoV2 infected LoM human lungs (red, $p<0.05$ ). The pathway enrichment score is shown on the $x$-axis.

Heatmaps illustrating the expression of human $c$, interferon genes and d, cytokine/chemokine genes in human lung tissue collected analyzed from SARS-CoV-2 infected LoM days $2(n=2), 6(n=3)$, and $14(n=3)$ post- exposure and naïve LoMs $(n=4)$. Color scale indicates the mean log10 transcripts per million (TPM).

a

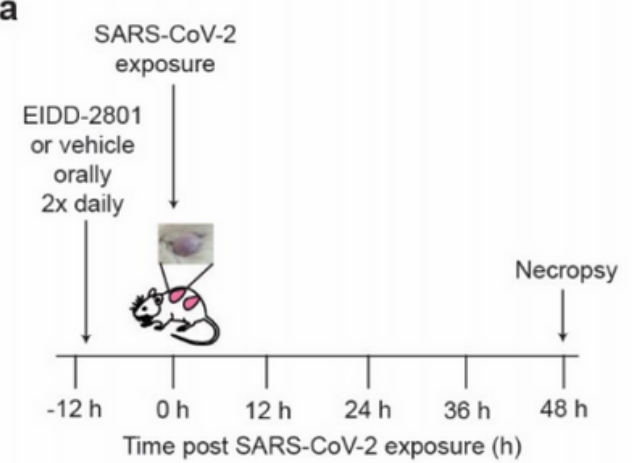

d

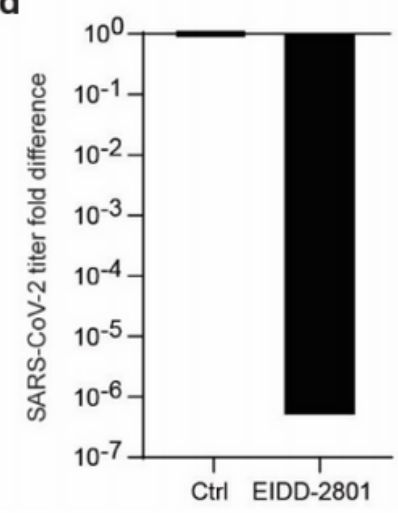

e b
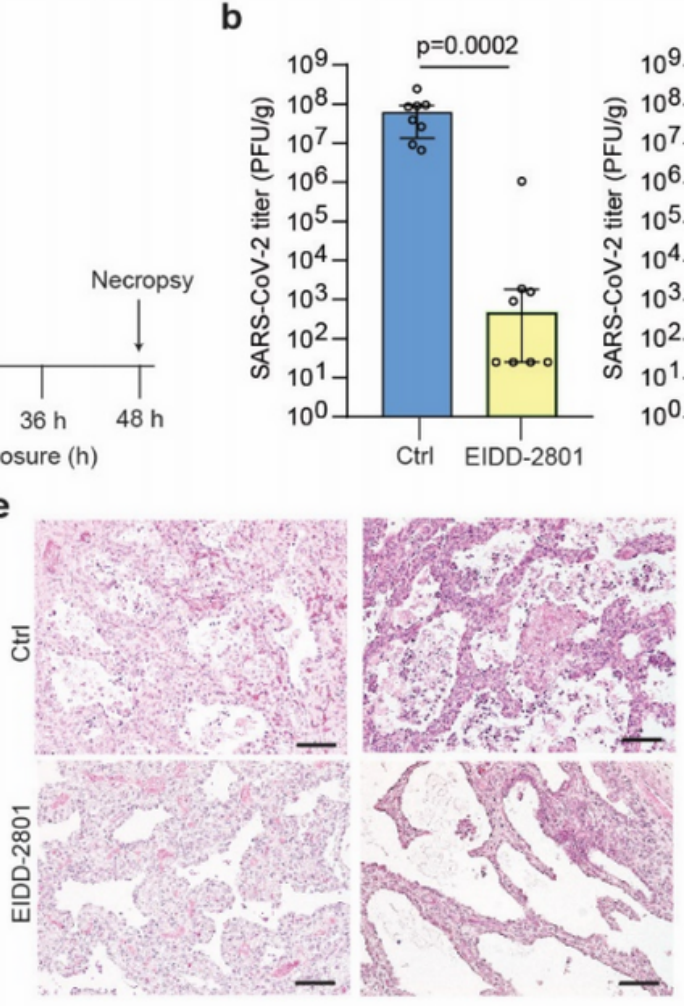

c

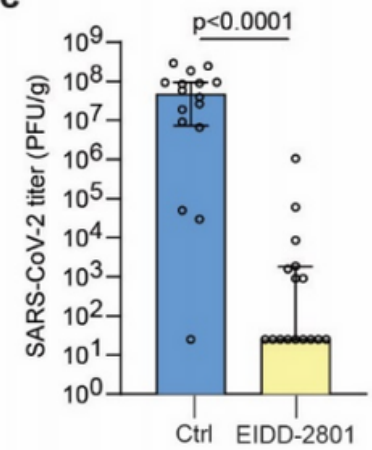

f

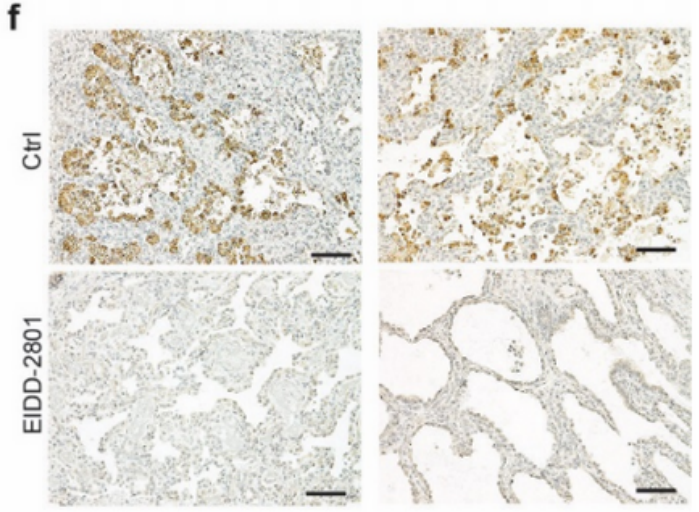

Figure 4

Pre-exposure prophylaxis with EIDD-2801, a broad-spectrum anti-coronavirus drug, potently prevents SARS-CoV-2 infection in vivo. a, Experimental design. LoM were administered EIDD-2801 or vehicle control $12 \mathrm{~h}$ prior to SARS-CoV- 2 exposure and every $12 \mathrm{~h}$ thereafter. Virus titers in human lung tissues were measured 2 days post- exposure. b and c, SARS-CoV-2 titers in the human lung tissue of LoM administered EIDD-2801 ( $\mathrm{n}=8$ per experiment, yellow) or control vehicle (Ctrl, $\mathrm{n}=8$ per experiment, blue) at 2 days post-exposure in two independent experiments shown b, separately and c, combined. Titers were compared with a two-tailed Mann-Whitney $U$ test. Horizontal and vertical lines represent the median and interquartile range respectively. $d$, Fold difference in SARS-CoV-2 titers in the human lung tissue of LoM relative to vehicle controls. e, H\&E staining and f, immunohistochemical staining for virus nucleoprotein 
(positive cells, brown) of human lung tissue of LoM administered EIDD-2801 ( $\mathrm{n}=8$ analyzed) or control vehicle (Ctrl, $n=8$ analyzed) at 2 days post-exposure (scale bars, 100 um).

\section{Supplementary Files}

This is a list of supplementary files associated with this preprint. Click to download.

- SupplementaryTable1Geneexpressionalltimepoints.xlsx

- SupplementaryTable2FoldChangeGeneexpressionalltimepoints.xlsx 